\title{
MMP-9, homocysteine and CRP circulating levels are associated with intraluminal thrombus thickness of abdominal aortic aneurysms - new implication of the old biomarkers
}

\author{
Ireneusz Wiernicki ${ }^{a, *}$, Barbara Millo $^{\mathrm{b}}, \mathrm{Krzysztof} \mathrm{Safranow}^{\mathrm{c}}$, Barbara Gorecka-Szyld $^{\mathrm{d}}$ and \\ Piotr Gutowski ${ }^{\mathrm{a}}$ \\ ${ }^{a}$ Department of Vascular Surgery and Angiology, Pomeranian Medical University, Szczecin, Poland \\ ${ }^{\mathrm{b}}$ Department of Clinical Biochemistry and Laboratory Diagnostics, Pomeranian Medical University, Szczecin, \\ Poland \\ ${ }^{\mathrm{c}}$ Department of Biochemistry and Medical Chemistry, Pomeranian Medical University, Szczecin, Poland \\ ${ }^{\mathrm{d}}$ Department of Radiology, Pomeranian Medical University, Szczecin, Poland
}

\begin{abstract}
Background: Abdominal aortic aneurysms (AAAs) are characterized by presence of high proteolytic activity, atherosclerotic lesions, extensive transmural inflammation and the presence of variably sized and shaped intraluminal thrombus (ILT). Therefore, we evaluated a possible association between plasma matrix metalloproteinase-9 (MMP-9), homocysteine (Hcy), high-sensitivity C-reactive protein (hsCRP) levels and ILT thickness in patients with AAA.

Methods: Plasma concentrations of MMP-9, Hcy and hsCRP were determined and ILT thickness was measured in 71 patients with AAA. They were divided into 2 groups according to ILT thickness: 34 patients with ILT mean thickness $\geqslant 9 \mathrm{~mm}$ and 37 patients with ILT $<9 \mathrm{~mm}$.

Results: Plasma MMP-9 and CRP concentrations in patients with thin ILT were significantly higher than in group with thick ILT (medians 610 vs. $485 \mathrm{ng} / \mathrm{mL}, p=0.00003$, and 7.7 vs. $3.3 \mathrm{mg} / \mathrm{L}, p<0.00001$, respectively). In contrast, plasma Hcy concentrations in patients with thin ILT were significantly lower than in the group with thick ILT (medians $14.3 \mathrm{vs} .19 .2 \mu \mathrm{mol} / \mathrm{L}$, $p<0.00001$ ). Multiple regression models adjusted for age and AAA diameter showed that thin ILT is an independent predictor of high MMP-9 and CRP concentrations, while thick ILT predicts high Hcy concentrations.

Conclusions: Association of higher plasma levels of MMP-9 and CRP with thin ILT may be related to two phenomena: thin thrombi convey more elastolysis-stimulating factors from blood to the AAA wall and thin thrombi convey more factors involved in proteolysis and inflammation from AAA wall to blood. The association of thin ILT with lower plasma Hcy concentrations may be related to the role of Hcy as a prothrombotic marker and needs further research.
\end{abstract}

Keywords: Abdominal aortic aneurysm, intraluminal thrombus, plasma biomarkers

\section{Introduction}

Since abdominal aortic aneurysms (AAAs) are usually asymptomatic, the present clinical challenge is to

* Address for correspondence: Ireneusz Wiernicki, Department of Vascular Surgery and Angiology, Powstancow Wielkopolskich 72, 70-111 Szczecin, Poland. Tel.: +48 9146611 56; Fax: +48 91466 11 57; E-mail: irekwie@wp.eu. diagnose them at an early stage, and to decipher the biological mechanisms responsible for the progressive dilatation and final rupture, in order to develop new diagnostic approaches [1]. Intraluminal thrombus (ILT) thickness seems to be an important local factor, which contributes to an individual's risk of aneurysm progression and rupture [2]. The average ILT thickness may be associated with circulating biomarkers which could play a role in the diagnosis of AAA and may be useful 
for predicting subsequent progression of AAA. Currently there are no adequate methods to identify AAAs at risk of progression and rupture. Degradation of extracellular matrix, especially elastin, within the aortic wall is a hallmark of AAAs. Among matrix-degrading enzymes, circulating matrix metalloproteinase-9 (MMP9) has been investigated most frequently [3]. High concentrations of MMP-9 have been observed to predict rapid coronary artery narrowing, ischemic heart disease incidence, AAA expansion, worse outcome in stroke patients, and cardiovascular death. The circulating MMP-9 level is considered to reflect the biological behavior of the aneurysm. The increased activity of elastase and MMP-9, which induce elastolysis, play a pivotal role in the etiology of AAAs. Plasma MMP9 concentration is significantly associated with AAA size and expansion, while concentrations of MMP-2 and MMPs inhibitors (TIMP 1 and 2), as well as the ratio (total MMP/total TIMP) did not predict size or expansion [4].

In AAA, MMP-9 expression in inflammatory cells is higher than in smooth muscle cells (SMCs), whereas MMP-2 demonstrates higher expression in SMCs than in inflammatory cells [5]. Leukocyte-derived MMP-9 is associated with aortic wall degeneration and aneurysm formation [6]. Immunohistochemistry localized MMP-9 to the interface between the thrombus and the underlying AAA wall and its expression was colocalized with the presence of neutrophils [7]. Moreover, the liquid interface between the thrombus and the wall also contained active MMP-9 [8]. MMP-9 plasma concentrations in patients with AAAs appeared to directly reflect the amount of MMP-9 released within aneurysm tissue. Serum MMP-9 concentration has been shown to fall significantly after AAA repair $[9,10]$. The first study reporting the prognostic value of MMP-9 in AAA therapy was published by Sangiorgi et al. [11].

In vitro evidence has demonstrated the induction of a serine elastase by homocysteine (Hcy) in vascular SMCs. Studies in animal models demonstrated that hyperhomocysteinemia could induce significant remodeling of the extracellular matrix of the arterial wall by inducing elastolysis through the activation of metalloproteinases [12]. High levels of Hcy are present in plasma of patients with AAA and the concentrations are positively correlated with AAA diameter $[13,14]$.

Atheromatous plaques, failed venous coronary bypasses and aneurysmal aortas produce C-reactive protein (CRP), thus illustrating that the production of CRP may represent a universal response to vascular injury [15]. In a recent study of a rabbit AAA model, increased serum hsCRP levels during AAA formation derive from aneurysmal arteries with degradation of elastin fibres [16].

Most AAAs contain ILT. Imaging features suggestive of instability or impending rupture include not only increased aneurysm size but also a low thrombus-tolumen ratio [17]. The thin ILT facilitates penetration of blood cells and plasma factors from the lumen towards the aneurysmal wall [18]. Other investigators observed from computed tomography (CT) images that ILT was significantly thinner in ruptured that in non-ruptured aneurysms (mean ILT thickness 9 vs. $19 \mathrm{~mm}$, respectively) [19]. It seems that thin ILT may play an important role in the increase of the AAA wall degradation and growth rate. In order to make further improvements in AAA monitoring, development of additional predictive tools other than AAA size alone is needed.

The aim of this study was to investigate the association of three plasma biomarkers (MMP-9, CRP, Hcy) of AAA progression with the thickness of ILT. These markers were chosen because their involvement in various mechanisms related to AAA formation and progression (Hcy - activation of elastolytic enzymes, MMP-9 - proteolysis, CRP - inflammation) has been reported.

\section{Methods}

\subsection{Subjects and study design}

The study comprised 71 consecutive patients (68 males and 3 females) with the diagnosed AAA monitored in the Department of the Vascular Surgery, Pomeranian Medical University. All the imaging of AAA was performed by computed tomography. Based on the mean ILT thickness all AAA patients were divided in 2 groups: 34 patients with the mean ILT thickness $\geqslant 9 \mathrm{~mm}$ and 37 patients with the mean ILT thickness $<$ $9 \mathrm{~mm}$. The $9 \mathrm{~mm}$ cut-off value was chosen because it is close to median ILT thickness for all patients $(8.8 \mathrm{~mm})$.

In order to establish the mean ILT thickness, measurements in the cross-section were performed in each $1 \mathrm{~cm}$ slice of overall length of AAA at " 12 " and " 6 " hours for anterior and posterior wall, respectively, and at "9" and " 3 " hours for lateral walls (Fig. 1). In our study, the linear distance from the lumen, through ILT to internal surface of AAA wall was measured because it is much more important than thrombus volume. Previous history and laboratory studies excluded coexisting renal disease or renal failure. The analysis of co- 
Table 1

Comparison of clinical, morphological and biochemical features between the groups stratified according to ILT thickness

\begin{tabular}{lccc}
\hline Features & ILT $<9 \mathrm{~mm}(n=37)$ & ILT $\geqslant 9 \mathrm{~mm}(n=34)$ & p-value $^{\mathrm{a}}$ \\
\hline Age [years] & $69(9)$ & $72(13)$ & 0.092 \\
Ischaemic heart disease & $14(38 \%)$ & $20(59 \%)$ & 0.10 \\
Past myocardial infarction & $3(8 \%)$ & $5(15 \%)$ & 0.47 \\
Past stroke & $0(0 \%)$ & $2(6 \%)$ & 0.23 \\
Arterial hypertension & $17(46 \%)$ & $21(62 \%)$ & 0.24 \\
Diabetes & $2(5 \%)$ & $3(9 \%)$ & 0.67 \\
Active smokers & $27(73 \%)$ & $30(88 \%)$ & 0.14 \\
ILT thickness [mm] & $4.8(4.5)$ & $10(4.2)$ & $<0.00001$ \\
AAA max. diameter [mm] & $40(9)$ & $54.5(14)$ & $<0.00001$ \\
MMP-9 [ng/mL] & $610(205)$ & $485(152)$ & 0.00003 \\
Hcy $[\mu \mathrm{mol} / \mathrm{L}]$ & $14.3(2.8)$ & $19.2(6.3)$ & $<0.00001$ \\
CRP [mg/L] & $7.7(3.4)$ & $3.3(1.7)$ & $<0.00001$ \\
\hline
\end{tabular}

Median (interquartile range) is presented for quantitative variables and number (\%) for qualitative variables;

${ }^{a}$ Mann-Whitney test for quantitative variables and Fisher exact test for qualitative variables.

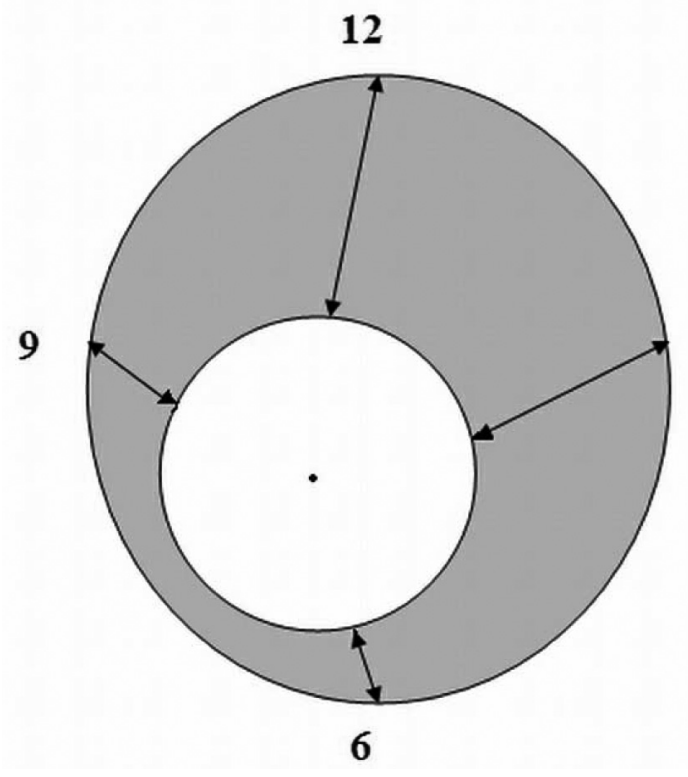

Fig. 1. The ILT measurements in the cross-section were performed in the presented four points in each $1 \mathrm{~cm}$ slice of overall length of AAA.

existing diseases and known cardiovascular risk factors did not reveal any statistically significant differences between the groups (Table 1). The studied patients were not administered any drugs that might increase Hcy concentration. The study protocol has been accepted by the Bioethical Committee of the Pomeranian Medical University.

\subsection{Measurements}

Peripheral venous blood was sampled in fasting patients and centrifuged within 1 hour with plasma samples subsequently stored at $-20^{\circ} \mathrm{C}$. High sensitivity CRP and total MMP-9 were measured using the sandwich enzyme immunoassay tests: CRP (EUROIMMUN ELISA, Medizinische Labordiagnostika AG, Lübeck, Germany) and MMP-9 (R\&D Systems, Minneapolis, USA). The detection limit for hsCRP was $0.8 \mathrm{ng} / \mathrm{mL}$ and intra-assay precision $(\mathrm{CV})$ was $5.0-5.8 \%$. The detection limit for MMP-9 was $0.156 \mathrm{ng} / \mathrm{mL}$. The intra-assay precision $(\mathrm{CV})$ was $1.9-$ $2.9 \%$. Total Hcy levels were measured using high performance liquid chromatography with fluorescence detection and kits from Bio-Rad, Germany. The coefficient of variation $(\mathrm{CV})$ was $2.7-4.4 \%$.

\subsection{Statistical methods}

Statistical analysis was performed using the Statistica 7.1 software (StatSoft, USA). Since distributions of most variables were significantly different from normal (Shapiro-Wilk test), the results were presented as median (interquartile range). Data were compared between groups with Mann-Whitney test for quantitative variables and with Fisher exact test for the qualitative ones. The Spearman rank correlation coefficient Rs was calculated to measure associations between quantitative variables. Multiple linear regression models including patients' age, AAA maximal diameter and presence of thin or thick ILT $(<9 \mathrm{~mm}$ vs. $\geqslant 9 \mathrm{~mm})$ as independent variables were used to identify independent predictors of MMP-9, CRP and Hcy concentrations as dependent variables. Variables with log-normal distributions (AAA diameter, CRP and Hcy) were transformed logarithmically before inclusion into the models. A $p$-value $<0.05$ was considered significant. 


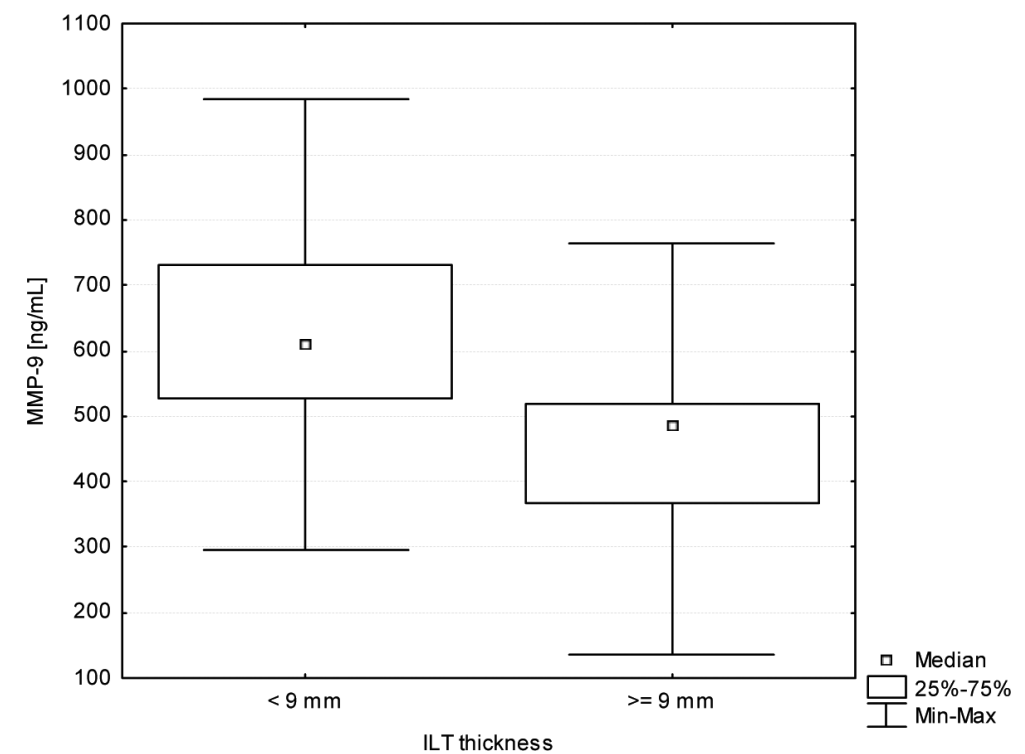

Fig. 2. Comparison of plasma MMP-9 concentrations between patients stratified according to ILT thickness.

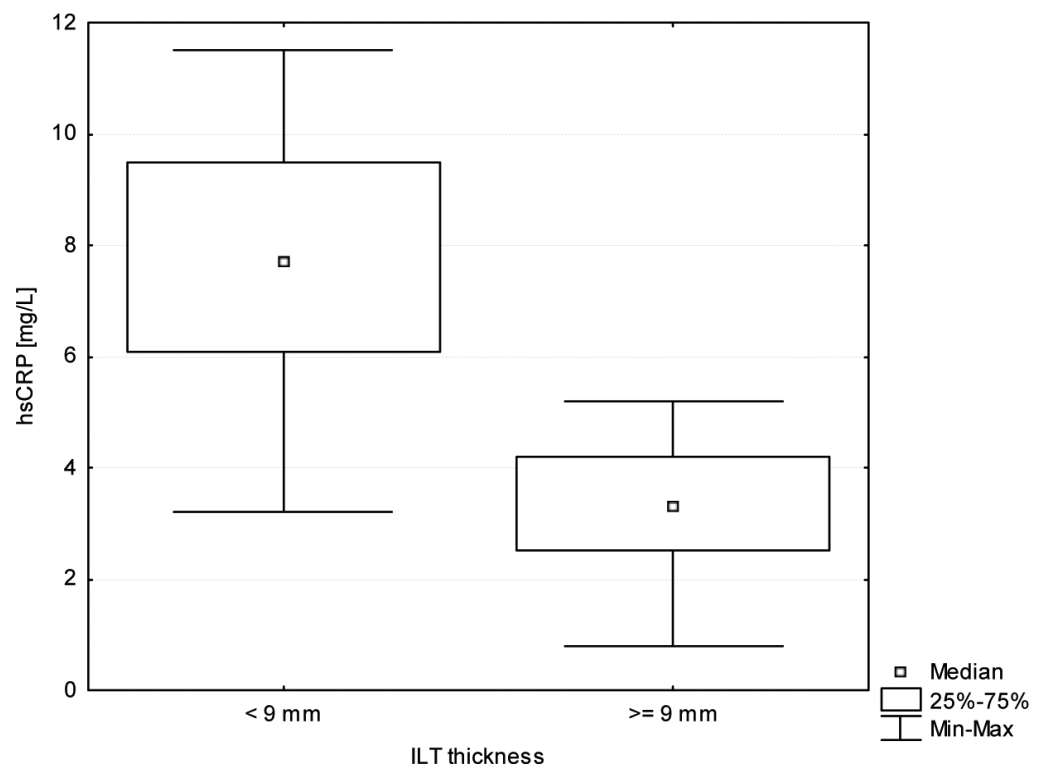

Fig. 3. Comparison of plasma hsCRP concentrations between patients stratified according to ILT thickness.

\section{Results}

Mean thickness of the ILT, maximal AAA diameter and the studied biochemical parameters in both groups are shown in the Table 1. Plasma MMP-9 levels were significantly higher in AAA patients with the mean ILT thickness $<9 \mathrm{~mm}$ than in patients with ILT $\geqslant 9 \mathrm{~mm}$ (Fig. 2). Plasma hs-CRP levels were also significantly higher in AAA patients with the mean ILT thickness $<9 \mathrm{~mm}$ than in those with ILT $\geqslant 9 \mathrm{~mm}$ (Fig. 3). In contrast, plasma Hcy concentrations were significantly lower in AAA patients with the mean ILT thickness $<$ $9 \mathrm{~mm}$ than in patients with ILT $\geqslant 9 \mathrm{~mm}$ (Fig. 4).

Table 2 presents correlations between morphological and biochemical parameters in the whole group of patients. ILT thickness strongly positively correlated with the maximal aneurysm diameter $(\mathrm{Rs}=+0.84$, $p<0.00001$, Fig. 5).

Both ILT thickness and AAA diameter correlated negatively with MMP-9 and CRP but positively with 
Table 2

Correlations between ILT thickness, AAA maximal diameter and MMP-9, CRP and Hcy plasma concentrations in the whole group of patients $(n=71)$

\begin{tabular}{lcccc}
\hline Correlated parameters & AAA diameter & MMP-9 & CRP & Hcy \\
\hline ILT thickness & +0.84 & -0.36 & -0.72 & +0.61 \\
& $<0.00001$ & 0.0018 & $<0.00001$ & $<0.00001$ \\
AAA diameter & - & -0.31 & -0.59 & +0.42 \\
& - & 0.0076 & $<0.00001$ & 0.00025 \\
MMP-9 & - & - & +0.49 & -0.27 \\
& - & - & 0.00001 & 0.023 \\
CRP & & & - & -0.55 \\
& & & & $<0.00001$ \\
\hline
\end{tabular}

Spearman rank correlation coefficients (Rs) and corresponding p-values are presented.

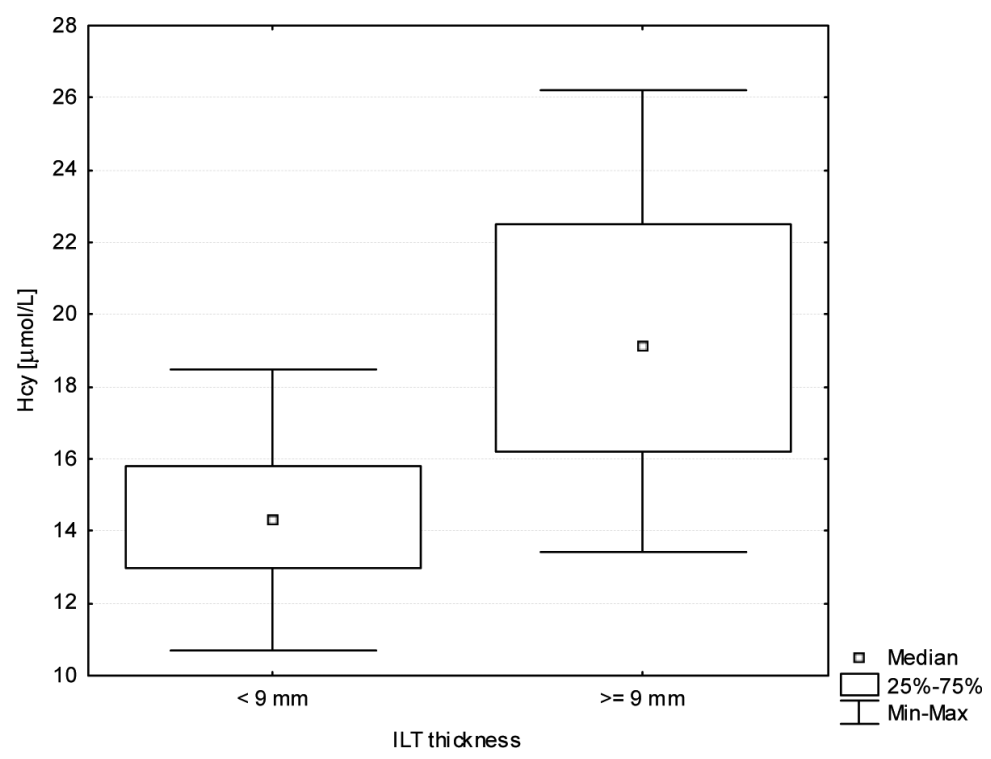

Fig. 4. Comparison of plasma Hcy concentrations between patients stratified according to ILT thickness.

Hcy; it is however worth noting that the correlations were stronger for ILT thickness. MMP-9 and CRP concentrations correlated positively with each other, and both correlated negatively with Hcy.

Multiple linear regression models adjusted for age and AAA diameter showed that presence of thin ILT $(<9 \mathrm{~mm})$ is an independent factor associated with higher MMP-9 $(\beta=+0.48, p=0.0010)$, higher CRP $(\beta=+0.66, p<0.00001)$ and lower Hcy $(\beta=-0.69$, $p<0.00001)$ plasma concentrations. Age and AAA diameter were not statistically significant predictors of the plasma markers in any of these models (data not shown).

\section{Discussion}

There is a growing number of animal and human association studies which have identified markers and strategies of potential value in improving identification, monitoring and treatment of AAA. In the present study we found some novel associations of plasma markers with ILT thickness which may be important for the understanding of pathophysiology of AAA.

Dobrin et al. reported that the intima and inner media of the AAA wall are supplied by the blood from its lumen [20]. ILT may be a significant barrier for the transport of various molecules from plasma through the walls of AAA [21]. A continuous network of canaliculi containing, among others, circulating neutrophils and macrophages, has been shown in ILT. However, cellular penetration from the AAA lumen occurs up to $10 \mathrm{~mm}$ from the luminal surface of the thrombus which indicates restriction of the cellular passage to the wall dependent on the thickness of ILT [18]. The MR imaging, histological and immunohistochemical studies of human thrombi sections showed that neutrophils 


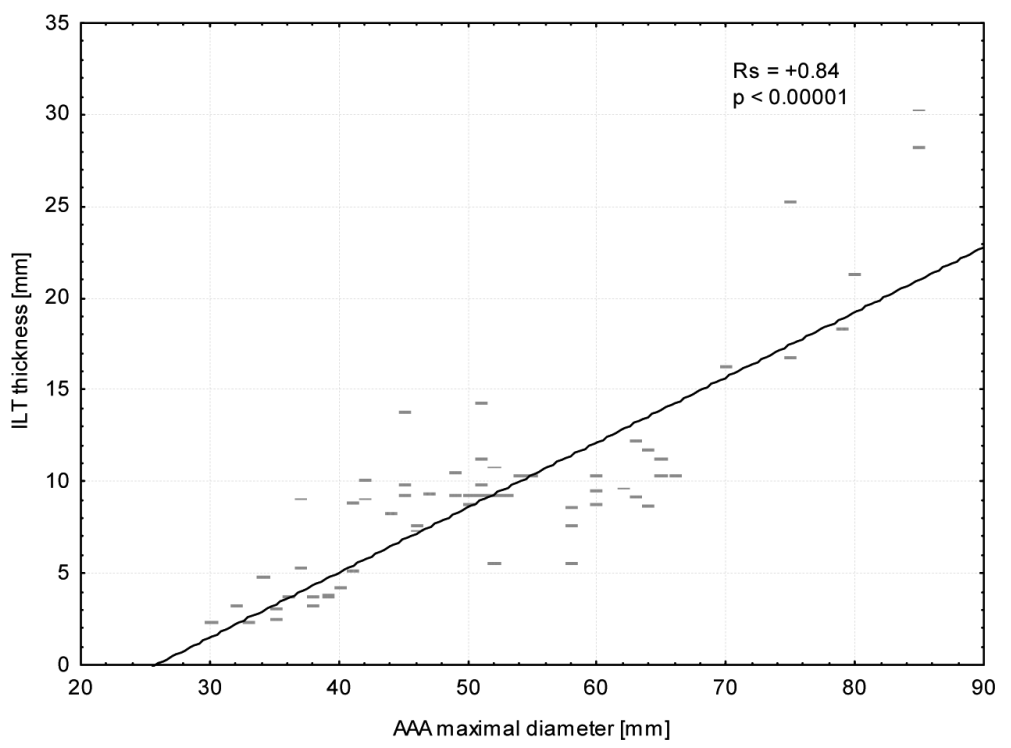

Fig. 5. The relationship between maximal AAA diameter and ILT thickness in all studied patients.

were localized within the luminal part of the thrombus. The luminal layer of ILT contained many neutrophils, which released various proteases, including pro-matrix metalloproteinase-9 (pro-MMP-9). Leukocyte elastase was also stored and released by the luminal ILT layer [22-24]. Aneurysmal tissue elastase activity is localized to the intima and inner media [25].

In this study plasma MMP-9 and CRP concentrations were significantly higher in patients with the average ILT thickness less than $9 \mathrm{~mm}$. This observation is novel and suggests that the AAA patients with the thin mural thrombus may show enhanced matrix-degrading proteolytic activity within the aneurysm wall and subsequently release higher amounts of proteolysis and inflammation markers to the blood. In our previous studies we documented that the wall of AAA covered by thin thrombi was characterized by reduced amount and irregular orientation of elastin fibres [26] and also by increased tissue activity of elastase and MMP-9 [2].

CRP represents the most extensively studied proinflammatory molecule, which is synthesized by hepatocytes. However, this protein may be also produced locally in vascular SMCs and macrophages. Moreover, CRP induced production of MMP-9 in human mononuclear cells in a concentration-dependent manner [27, 28]. This mechanism may be an explanation of the strong positive correlation between CRP and MMP-9 plasma concentrations in our AAA patients.

Elevated plasma CRP in patients with symptomatic aortic aneurysm is an independent predictor of poor prognosis. CRP levels higher than $6.3 \mathrm{mg} / \mathrm{L}$ are as- sociated with high risk for short-term mortality [29]. A significant elevation of CRP was found in patients who presented symptoms or rupture of an AAA [30]. It seems that thin ILT is associated with high plasma CRP, and both features are risk factors of AAA rupture. Further prospective studies are needed to elucidate the cause-and-effect relationships between ILT thickness, CRP and clinical outcome.

Since atherosclerosis of aorta is associated with AAA development, patients with AAA usually have advanced atherosclerotic lesions also in other arteries. According to the current American Heart Association guidelines AAA is considered an atherosclerotic equivalent. The tissues responsible for homocysteine production and export to the plasma are not well known. However, a significant role of the liver in the regulation of plasma Hcy levels is considered. Elevated plasma homocysteine is a risk factor for atherosclerotic disease, but Hcy can also stimulate elastolysis in the arterial media via activation of elastase and matrix metalloproteinases. AAA patients have significantly higher plasma Hcy than healthy subjects [31]. In our study Hcy levels correlated positively with AAA diameter. AAA growth rate among patients with hyperhomocysteinemia $(>15 \mu \mathrm{mol} / \mathrm{L})$ was double that of patients with normal Hcy [32]. However, Hcy levels were not associated with AAA tissue inflammation [33].

In our AAA patients correlation of ILT thickness with Hcy was positive, conversely to the negative correlation of MMP-9 and CRP. It may be related to the fact that AAA wall cells produce MMP-9 and CRP (which 
are conveyed through thin ILT to blood) but not Hcy. Therefore, thick ILT cannot decrease plasma Hcy by inhibiting its transport from AAA wall to blood. However, hyperhomocysteinemia may play some role in the development of ILT in the sac of aneurysm. In experimental and clinical studies, a homocysteine-mediated oxidant stress has been shown to trigger platelet activation leading to arterial thrombosis in patients with severe hyperhomocysteinemia, and hyperhomocysteinemia is also related to impaired fibrinolysis [34]. It is possible that higher Hcy in patients with thick thrombi reflects the individual susceptibility to faster ILT growth due to some prothrombotic mechanisms, which need further research.

In conclusion, association of higher plasma levels of MMP-9 and CRP with thin ILT may be related to two phenomena: thin thrombi convey more elastolysisstimulating factors from blood to the AAA wall and thin thrombi convey more factors involved in proteolysis and inflammation from AAA wall to blood. In contrast, thicker ILT constitutes a significant barrier for proteolytic and inflammatory factors. Association of thin ILT with lower plasma Hcy concentrations may be related to the role of Hcy as a prothrombotic marker and needs further research.

In order to further improve clinical management of patients with AAA, development of additional predictive tools other than aneurysm size alone is needed. Non-invasive risk assessment of AAA progression and rupture by determination of plasma biomarker levels may play an important role in the future clinical practice, improving selection criteria for vascular intervention. However, prospective assessment in large multicenter cohorts will be needed to confirm the potential role of multiple circulating biomarkers in the prediction of AAA progression.

\section{References}

[1] J.B. Michel, J.L. Martin-Ventura, J. Egido, N. Sakalihasan, V. Treska, J. Lindholt et al., Novel aspects of the pathogenesis of aneurysms of the abdominal aorta in humans, Cardiovasc Res 90 (2010), 18-27.

[2] I. Wiernicki, E. Stachowska, K. Safranow, M. Cnotliwy, M. Rybicka, M. Kaczmarczyk et al., Enhanced matrix-degrading proteolytic activity within the thin thrombus-covered wall of human abdominal aortic aneurysms, Atherosclerosis 212 (2010), 161-165.

[3] J. Golledge, P.S. Tsao, R.L. Dalman and P.E. Norman, Circulating markers of abdominal aortic aneurysm presence and progression, Circulation 118 (2008), 2382-2392.
[4] J.S. Lindholt, S. Vammen, H. Fasting, E.W. Henneberg and L. Heickendorff, The plasma level of matrix metalloproteinase 9 may predict the natural history of small abdominal aortic aneurysms. A preliminary study, Eur J Vasc Endovasc Surg 20 (2000), 281-285.

[5] V. Lesauskaite, M.C. Epistolato, M. Castagnini, S. Urbonavicius and P. Tanganelli, Expression of matrix metalloproteinases, their tissue inhibitors, and osteopontin in the wall of thoracic and abdominal aortas with dilatative pathology, Hum Pathol 37 (2006), 1076-1084.

[6] S. Saito, N. Zempo, A. Yamashita, H. Takenaka, K. Fujioka and K. Esato, Matrix metalloproteinase expressions in arteriosclerotic aneurysmal disease, Vasc Endovascular Surg 36 (2002), 1-7.

[7] M. Kazi, C. Zhu, J. Roy, G. Paulsson-Berne, A. Hamsten, J. Swedenborg et al., Difference in matrix-degrading protease expression and activity between thrombus-free and thrombuscovered wall of abdominal aortic aneurysm, Arterioscler Thromb Vasc Biol 25 (2005), 1341-1346.

[8] V. Fontaine, M.P. Jacob, X. Houard, P. Rossignol, D. Plissonnier, E. Angles-Cano et al., Involvement of the mural thrombus as a site of protease release and activation in human aortic aneurysms, Am J Pathol 161 (2002), 1701-1710.

[9] D.M. Hovsepian, S.J. Ziporin, M.K. Sakurai, J.K. Lee, J.A. Curci and R.W. Thompson, Elevated plasma levels of matrix metalloproteinase-9 in patients with abdominal aortic aneurysms: a circulating marker of degenerative aneurysm disease, J Vasc Interv Radiol 11 (2000), 1345-1352.

[10] T. Watanabe, A. Sato, T. Sawai, M. Uzuki, H. Goto, H. Yamashita et al., The elevated level of circulating matrix metalloproteinase- 9 in patients with abdominal aortic aneurysms decreased to levels equal to those of healthy controls after an aortic repair, Ann Vasc Surg 20 (2006), 317-321.

[11] G. Sangiorgi, R. D’Averio, A. Mauriello, M. Bondio, M. Pontillo, S. Castelvecchio et al., Plasma levels of metalloproteinases-3 and -9 as markers of successful abdominal aortic aneurysm exclusion after endovascular graft treatment, Circulation 104 (2001), I288-295.

[12] B. Giusti, R. Marcucci, I. Lapini, I. Sestini, M. Lenti, M. Yacoub et al., Role of hyperhomocysteinemia in aortic disease, Cell Mol Biol 50 (2004), 945-952.

[13] F. Sofi, R. Marcucci, B. Giusti, G. Pratesi, B. Lari, I. Sestini et al., High levels of homocysteine, lipoprotein (a) and plasminogen activator inhibitor-1 are present in patients with abdominal aortic aneurysm, Thromb Haemost 94 (2005), 1094-1098.

[14] T. Brunelli, D. Prisco, S. Fedi, A. Rogolino, A. Farsi, R. Marcucci et al., High prevalence of mild hyperhomocysteinemia in patients with abdominal aortic aneurysm, J Vasc Surg 32 (2000), 531-536.

[15] T. Vainas, F.R. Stassen, R. de Graaf, E.L. Twiss, S.B. Herngreen, R.J. Welten et al., C-reactive protein in peripheral arterial disease: relation to severity of the disease and to future cardiovascular events, J Vasc Surg 42 (2005), 243-251.

[16] G. Huang, A. Wang, X. Li, M. Long, Z. Du, C. Hu et al., Change in high-sensitive C-reactive protein during abdominal aortic aneurysm formation, J Hypertens 27 (2009), 1829_ 1837.

[17] D. Rakita, A. Newatia, J.J. Hines, D.N. Siegel and B. Friedman, Spectrum of CT findings in rupture and impending rupture of abdominal aortic aneurysms, Radiographics 27 (2007), 497-507.

[18] R. Adolph, D.A. Vorp, D. Steed, M.W. Webster, M.V. Kameneva and S.C. Watkins, Cellular content and permeability of 
intraluminal thrombus in abdominal aortic aneurysm, $J$ Vasc Surg 25 (1997), 916-926.

[19] T. Kushihashi, H. Munechika, S. Matsui and T. Moritani, CT of abdominal aortic aneurysms: aneurysmal size and thickness of intra-aneurysmal thrombus as risk factors of rupture, Nippon Igaku Hoshasen Gakkai Zasshi 51 (1991), 219-227.

[20] P.B. Dobrin, W.H. Baker and W.C. Gley, Elastolytic and collagenolytic studies of arteries, Arch Surg 119 (1984), 405-408.

[21] D.A. Vorp, W.J. Federspiel and M.W. Webster, Does laminated intraluminal thrombus within abdominal aortic aneurysm cause anoxia of the aortic wall? J Vasc Surg 23 (1996), 540541.

[22] A. Nchimi, O. Defawe, D. Brisbois, T.K. Broussaud, J.O. Defraigne, P. Magotteaux et al., MR imaging of iron phagocytosis in intraluminal thrombi of abdominal aortic aneurysms in humans, Radiology 254 (2010), 973-981.

[23] L. Sarda-Mantel, M. Coutard, F. Rouzet, O. Raguin, J.M. Vrigneaud, F. Hervatin et al., ${ }^{99 m}$ Tc-Annexin-V Functional Imaging of Luminal Thrombus Activity in Abdominal Aortic Aneurysms, Arterioscler Thromb Vasc Biol 26 (2006), 21532159.

[24] V. Fontaine, Z. Touat, el M. Mtairag, R. Vranckx, L. Louedec, $\mathrm{X}$. Houard et al., Role of leukocyte elastase in preventing cellular re-colonization of the mural thrombus, Am J Pathol 164 (2004), 2077-2087.

[25] R.W. Busuttil, H. Rinderbriecht, A. Flesher and C. Carmack, Elastase activity: the role of elastase in aortic aneurysm formation, J Surg Res 32 (1982), 214-217.

[26] I. Wiernicki, M. Cnotliwy, I. Baranowska-Bosiacka, E. Urasinska, A. Kwas, J. Bober et al., Elastin degradation within the abdominal aortic aneurysm wall - relationship between intramural $\mathrm{pH}$ and adjacent thrombus formation, Eur J Clin Invest 38 (2008), 883-887.
[27] A. Nabata, M. Kuroki, H. Ueba, S. Hashimoto, T. Umemoto, H. Wada et al., C-reactive protein induces endothelial cell apoptosis and matrix metalloproteinase- 9 production in human mononuclear cells: Implications for the destabilization of atherosclerotic plaque, Atherosclerosis 196 (2008), 129-135.

[28] U. Singh, M.R. Dasu, P.G. Yancey, A. Afify, S. Devaraj and I. Jialal, Human C-reactive protein promotes oxidized low density lipoprotein uptake and matrix metalloproteinase- 9 release in Wistar rats, J Lipid Res 49 (2008), 1015-1023.

[29] M. Schillinger, H. Domanovits, K. Bayegan, T. Holzenbein, M. Grabenwoger, J. Thoenissen et al., C-reactive protein and mortality in patients with acute aortic disease, Intensive Care Med 28 (2002), 740-745.

[30] H. Domanovits, M. Schillinger, M. Mullner, T. Holzenbein, K. Janata, K. Bayegan et al., Acute phase reactants in patients with abdominal aortic aneurysm, Atherosclerosis 163 (2002), 297-302.

[31] F. Sofi, R. Marcucci, B. Giusti, G. Pratesi, B. Lari, I. Sestini et al., High levels of homocysteine, lipoprotein (a) and plasminogen activator inhibitor-1 are present in patients with abdominal aortic aneurysm, Thromb Haemost 94 (2005), 1094-1098.

[32] K.J. Halazun, K.A. Bofkin, S. Asthana, C. Evans, M. Henderson and J.I. Spark, Hyperhomocysteinaemia is Associated with the Rate of Abdominal Aortic Aneurysm Expansion, Eur J Vasc Endovasc Surg 33 (2007), 391-394.

[33] V. Arapoglou, A. Kondi-Pafiti, D. Rizos, T. Kotsis, C. Kalkandis and K. Katsenis, The influence of total plasma homocysteine and traditional atherosclerotic risk factors on degree of abdominal aortic aneurysm tissue inflammation, Vasc Endovascular Surg 43 (2009), 473-479.

[34] M.N. Di Minno, E. Tremoli, A. Coppola, R. Lupoli and G. Di Minno, Homocysteine and arterial thrombosis: Challenge and opportunity. Thromb Haemost 103 (2010), 942-961. 


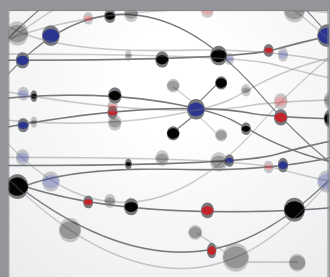

The Scientific World Journal
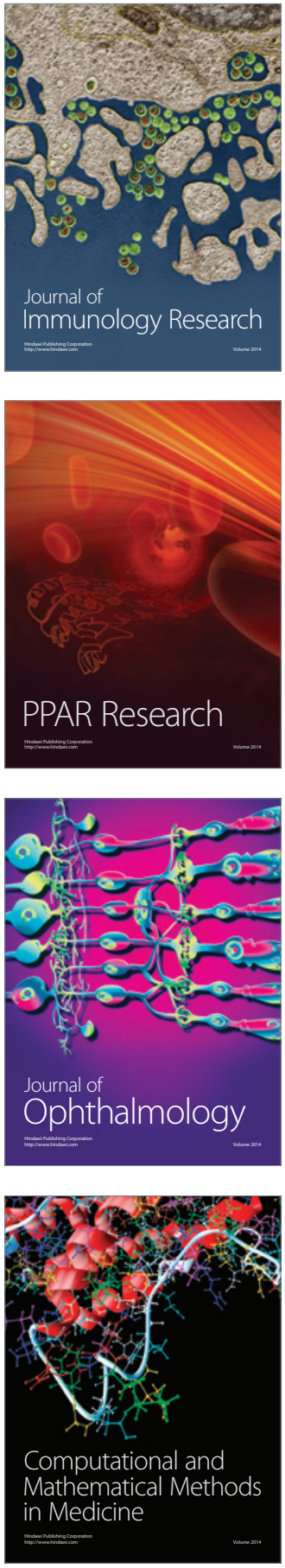

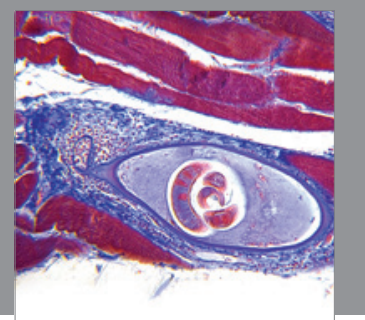

Gastroenterology

Research and Practice
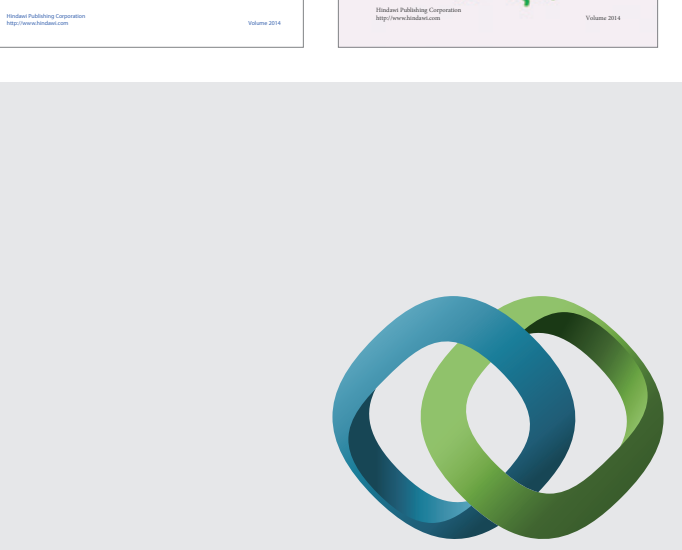

\section{Hindawi}

Submit your manuscripts at

http://www.hindawi.com
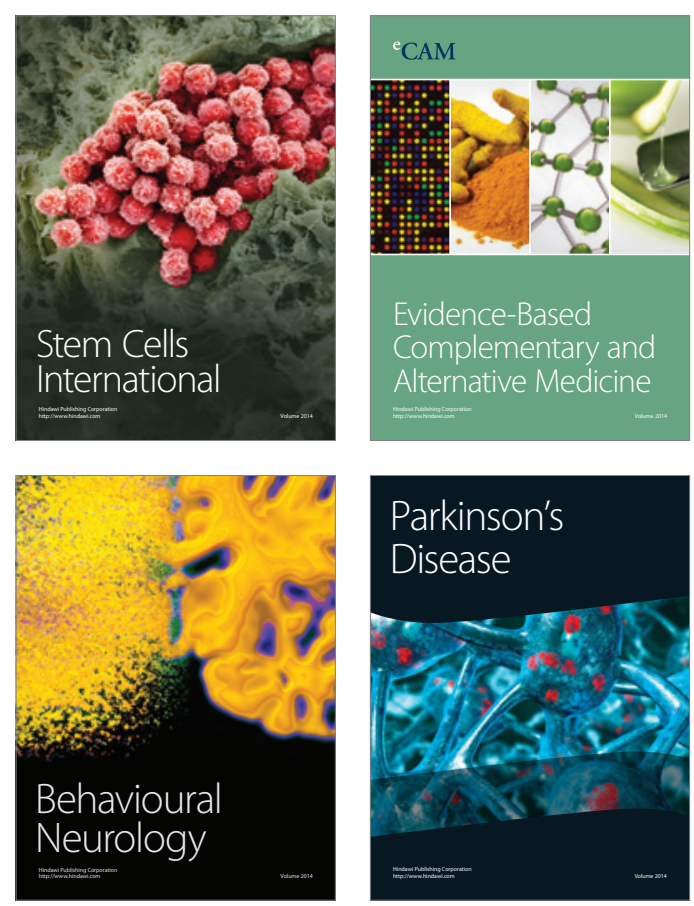

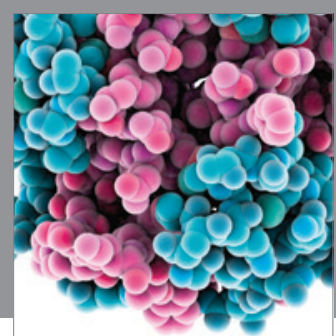

Journal of
Diabetes Research

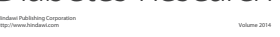

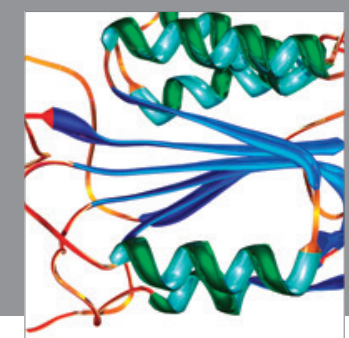

Disease Markers
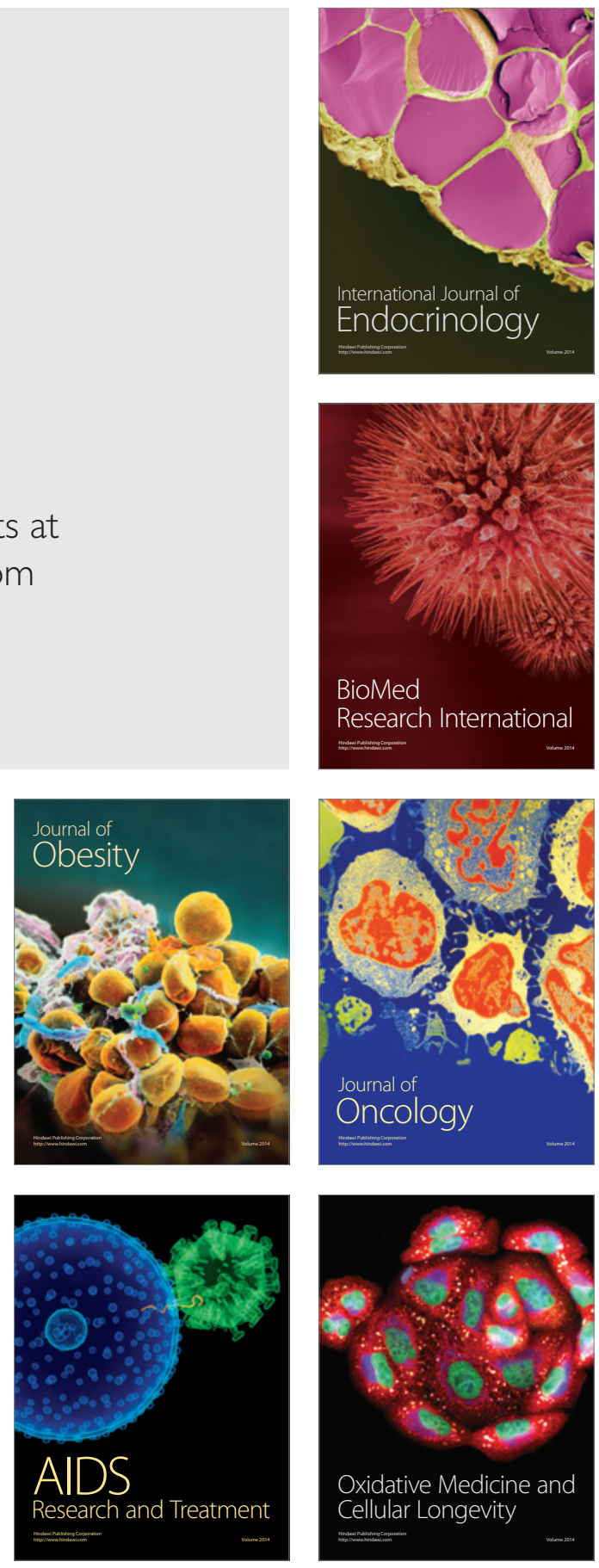